\title{
Estética universitaria: el placer por aprender es el placer por vivir ${ }^{1}$
}

\author{
University aesthetics: the pleasure to learn is the pleasure to live
}

\section{Raúl Alberto Henao Vélez ${ }^{2}$}

Henao V, Raúl A

miradas $N^{\circ} 2-2019$ ISSN digital N²539-3812 Págs 41 - 65

Recepción: Febrero 12 de 2019

Aprobación: Mayo 30 de 2019

Publicación: Junio 30 de 2019

\section{Resumen}

Este artículo presenta el proceso de investigación Estética universitaria en el cual, parte de la pregunta por el placer que produce o no el entorno estético del mundo universitario. El juicio estético de Kant es un referente teórico en tanto relaciona el juicio estético con el grado de placer. Así, los hallazgos de esta tesis demuestran que más allá de las coherencias entre las teorías estéticas de Kant y los datos, la pregunta por la estética, como placer, comprende un espectro que va más allá de lo visual y sufre una apertura que no lo hace depender exclusivamente de lo sensorial, con lo que comúnmente suele relacionarse, y, es, además, los buenos actos y la novedad presente en la acción creativa e intelectual.

La metodología relacional basada en la Semiótica y teoría triádica del Signo en Charles Peirce permitió inicialmente aglutinar el dato original, pasar a la interpretación, la abstracción, la proposición y finalmente a la conclusión final sin perder fidelidad entre el dato y la conclusión.

Los hallazgos muestran a un sujeto que se garantiza su propio placer procurando el de los demás. Cultiva favores estéticos en el otro que luego serán devueltos, esto, en relaciones estéticas dependientes, activas mediadas por el signo, y que comprende, el dar y recibir. Esto, dibuja una complejidad de relaciones estéticas donde nadie es independiente y por el contrario se presenta como un solo organismo dinámico y autónomo.

1 El presente artículo es producto de los resultados obtenidos en la investigación "Estética universitaria: El placer por aprender es el placer por vivir". En el marco de la Maestría en Comunicación Educativa de la Universidad tecnológica de Pereira.

2 Magister en Comunicación Educativa de la Universidad Tecnológica de Pereira. Catedrático de la facultad de comunicación Audiovisual del Politécnico Jaime Isaza Cadavid. Correo, rhenaovelez@gmail.com 
Palabras clave: Estética, placer, emoción, novedad, creatividad, bondad, amor, inteligencia.

\section{Abstract}

This article presents the research process Aesthetic university: the pleasure to learn is the pleasure to live, which, part of the question about the pleasure that produces or not the aesthetic environment of the university world. Kant's aesthetic judgment establishes an important theoretical reference insofar as it links the aesthetic judgment with the degree of pleasure it generates in the subject. Therefore, the findings of this thesis show that beyond the coherence between the aesthetic theories of Kant and the data, these last ones indicate that the aesthetic's matter, as a degree of pleasure, it includes a spectrum that goes beyond the visual and it suffers an opening that it doesn't depend exclusively from the sensory stimulation, with what is usually associated, but it's also sensitive to moral and intellectual stimulation. Thus, not only the beautiful generates esthetic pleasure, but also, the good actions and the novelty shown in the creative and intellectual act.

The findings show a subject who obtains and guarantees his own pleasure by obtaining it from others. Cultivate aesthetic favors in others that these will be returned later or immediately, this, in dependent and active aesthetic relations mediated by the sign, and that includes, giving and receiving. This draws a complexity of aesthetic relationships where no one is independent and, on the contrary, it appears as a dynamic and autonomous single organism.
The original question about the aesthetic stimulus in the university classroom and the data collected and interpreted shows an important conceptual transformation that highlights the importance of the Politécnico Jaime Isaza Cadavid's aesthetic experience to the world. Where, for seeking pleasure in the expanded sense of esthetics, is to make this world a more pleasant and kinder place to live.

Keywords: aesthetics, pleasure, emotion, novelty, creativity, goodness, love, intelligence

\section{Introducción}

El presente texto desarrolla la tesis: la carencia de belleza y agradabilidad presentes en los ambientes universitarios paraliza toda experiencia estética frente al aprendizaje. Esta afirmación nace de varias observaciones y lecturas: primera, es la ausencia motivacional que generan las actuales condiciones estéticas de las aulas de aprendizaje, en comparación con el alto grado de estetización y placer que brindan los espacios de consumo en nuestra sociedad contemporánea; la segunda, es la incoherencia discursiva entre la alta calidad educativa que debe tener nuestra sociedad frente a la realidad de nuestras aulas universitarias; la tercera, es la falta de placer presente en el trabajo educativo, lo que es herencia de una tradición católica que valora los logros en función de los sacrificios experimentados para su obtención.

Entre los tiempos medievales, origen de las formas de aprendizaje actuales y los tiempos modernos se ubica la figura 
de Kant y su desarrollo de la Crítica del juicio, donde él, da fundamento a los principios teóricos de la estética; allí, el autor plantea la relación directa entre lo agradable y lo bello, con el deseo y el placer. Desde la perspectiva disciplinar de la estética, Kant, argumenta una conexión directa entre el deseo y el placer, producto de una danza simultánea entre la imaginación y el entendimiento en la experiencia frente a lo bello. Este autor fue la fuente que guio la reflexión teórica.

En los tiempos actuales, reflexiones como las de Augustowsky (2010), dan cuenta de la preocupación enunciada:

En tal sentido Augustowsky (2010) declara,

En las paredes del aula, con la concepción de que a medida que uno tiene pensamiento formal necesita menos imágenes, que un dibujo es un asistente cuando uno no sabe leer. Es una idea que desconoce totalmente la complejidad de la información iconográfica (...) En los espacios universitarios lo que más valor tiene es la palabra y la palabra escrita (p. 19).

Consecuente con la anterior reflexión problemática, surgió la siguiente pregunta: ¿Cómo es la percepción que tiene el grupo de estudiantes y profesores del Politécnico Jaime Isaza Cadavid consultados, acerca de la estética y la creatividad y su relación con el aprendizaje?
La metodología relacional basada en la Semiótica y teoría triádica del signo en Charles Peirce permitió inicialmente aglutinar el dato original, pasar a la interpretación, la abstracción, la proposición y finalmente a la conclusión final sin perder fidelidad entre el dato y la conclusión. Se reunieron muestras de 15 personas, diez estudiantes y cinco profesores, cada uno con 20 preguntas que multiplicadas da un total de 350 las cuales, además, fueron cruzadas entre sí, elevando a un considerable número la cantidad de datos analizados. Estos datos, se aglutinaron y modularon en un proceso de reunión semántica de síntesis. Pero antes, permítame le invito a ver el siguiente video que describe los principales hallazgos de esta tesis. Link a video:

https://www.youtube.com/watch?v=7nyH1SJ0VcU\& feature $=$ youtu.be

Los resultados permitieron comprender la realidad y lo ideales estéticos de la comunidad educativa de la Facultad de Comunicación datada. De este modo, el trabajo procuró aportar elementos de análisis a la discusión acerca de la educación creativa y la estética, en el ámbito de las aulas universitarias.

Es necesario precisar que los hallazgos de esta investigación, al ser abordada desde la estética, pretenden brindar elementos de valoración para comprender el placer y la emoción presente en el aprendizaje. El placer y la emoción son fundamento para una educación que estimule la creatividad, no la productividad, ni la autoridad, ni la memoria. El enfoque estético no pretende hacer estudiantes más inteligentes, sino que procura formar 
estudiantes que encuentren desarrollo pleno de sus potencialidades de ser y hacer en el objeto de estudio.

Al final de este artículo se verá como aquello que inicio con una pregunta acerca de los estímulos placenteros del espacio académico y sus objetos, terminó refiriéndose a algo más amplio y complejo como lo son las interacciones con las personas. Se demostrará como el sujeto no sólo percibe estímulos estéticos, sino que los produce, en una estética dinámica y vital que une el sujeto, el objeto y el signo estético en uno solo. Esto, como una unidad donde la acción y la reacción de uno dependen, por un lado, de los estímulos de placer o displacer que percibe del otro, esto, en una reacción binaria, o, por otra parte, de reacciones planeadas y en ocasiones transformadas en la lógica racional del sujeto estableciendo una relación tríadica con el signo estético.

\section{Metodología}

Por primera y última vez en este artículo me expresaré en primera persona, no es capricho, es la necesidad de dejar claro que soy un artista, docente universitario que por mi sensibilidad y oficio docente terminé por preguntarme acerca de lo que nos estimula y genera un placer por aprender. El problema surgió cuando percibí un exceso de deber en la formación académica de los jóvenes y poco placer por aprender expresado en la actitud de hacer menos porque siempre hay mucho que hacer. Displicencia. ¿Es esa la herencia académica que hemos dejado en ellos? ¿El conocimiento, la cultura, la ciencia, la técnica como un deber de producir?
Graduarse rápido para salir a producir dinero no es motivación para un ser sensible. Eso pensé yo, en ese momento.

Pero al llegar a este punto, me enfrente a la duda siguiente, eran mis preconceptos los que guiaban la preocupación, los cuales se los colocaba a las personas, suponiendo que ellos sentían igual a mí, o podrían existir otras ideas frente a lo que yo pensaba. Esta inquietud, me llevo a construir una metodología cualitativa, que considerara la percepción de los otros, para tratar de comprender los sentidos que construían las personas alrededor de la belleza, el placer, las emociones, la imaginación, el conocimiento y hasta el amor. Para luego, contrastar dichas percepciones con las teorías acerca de dichos conceptos, para lo cual me basé en Kant.

La metodología partió de 20 preguntas formuladas a 15 personas que suman un total de 350 datos que cruzados entre si elevan aún más la cantidad de datos a una cifra considerable de análisis. Estos, indagaron por la experiencia estética de los estudiantes y docentes de la facultad de comunicación audiovisual del Politécnico Jaime Isaza Cadavid que respondió a éstas. Los interrogantes, se hicieron a 15 personas, 10 estudiantes de pregrado y cinco docentes. 


\section{Objetivos específicos Preguntas}

1. Analizar e interpretar las ideas y percepciones que las personas tienen acerca de: lo bello, lo sublime, la imaginación, la inteligencia, el amor y la reflexión.
¿En pocas palabras, qué entiende por lo bello? ¿En pocas palabras, qué entiende por lo estético?

¿En pocas palabras, qué entiende por lo sublime?

¿En pocas palabras, qué entiende por lo agradable?

¿Qué entiende por amor?
Las siguientes 10 preguntas buscaron comprender las relaciones que estas personas establecen con el placer, esto incluye las emociones y el amor;
2. Relacionar en las personas sus placeres con: lugares, personas, cosas y actividades.
¿Imagine tres lugares bellos y descríbalos?

¿Qué cosas, sentimientos, lugares, personas asocia con la palabra placer?

Resuma una emoción vivida.

¿En este escenario, puede identificar algo bello?

¿Qué elementos harían que usted se amañara más en las aulas de clase?

¿Explique si hay algo o alguien dentro del aula de clase que le genere emoción?

¿El color con que están pintadas las aulas de clase, qué sensación le generan?

¿Qué sensación le genera las aulas de clase actuales?

¿Cómo cree que las aulas de clase estimularían la imaginación?

¿De qué forma la belleza de alguien del salón ha sido un estímulo para ir a estudiar?

¿De qué manera involucra el placer en el estudio?

¿Qué es lo que más admira de sus compañeros y docentes?

¿Valora más la inteligencia o la belleza en un compañero(a) ? ¿Por qué?

¿Valora más la inteligencia o la belleza en una persona? ¿Por qué?

¿Por qué motivos, fuera de los académicos, ha ido usted a clase?

¿Describa si la admiración por alguien del salón ha sido un estímulo para ir a estudiar? 
En las últimas cinco se concentran los interrogantes en los conceptos e ideas que las personas tuvieron acerca de la imaginación y la creatividad.

\begin{tabular}{ll}
\hline & $\begin{array}{l}\text { ¿Qué entiende por creatividad? } \\
\text { ¿Cómo estimularía la } \\
\text { creatividad en alguien? }\end{array}$ \\
3. Comprender & ¿Describa algún acto creativo \\
lo que las & que haya experimentado? \\
personas & ¿Sabe qué lo pone creativo? \\
sienten y & ¿Imagine y describa un espacio \\
entienden & de aprendizaje creativo? \\
por & ¿Recuerde alguna clase \\
creatividad. & que le haya estimulado la \\
& creatividad? Descríbala. \\
& ¿Con sus palabras, diga un aula \\
& que estimulara su creatividad? \\
\hline
\end{tabular}

Las preguntas fueron un pretexto para iniciar la conversación con las personas, pero era consciente que lo importante no era la respuesta literal que daban, sino lo que se podía inferir de ella. Y para esto se hizo énfasis en el análisis de la relación de las 20 respuestas, pues de esta manera, se pudo agrupar a partir de elementos comunes, distintos, o emergentes.

\section{Datos}

Conceptos acerca de la belleza, lo sublime, lo agradable y el amor

El primer hallazgo acerca del concepto de belleza está ligado al juicio de la belleza física que pretende ser universal $\mathrm{y}$ objetiva y que encuentra, soporte cultural, en la belleza clásica, el número y la proporción. Concepto griego que lo encontramos además en Kant.
El segundo hallazgo acerca del concepto de belleza está ligado al juicio de la belleza como conjunto de cualidades interiores en una persona. Este concepto está ligado a la tradición religiosa cristiana de la belleza interior y en Kant aparece como conjunto de valores éticos.

El tercer hallazgo es de tipo semiótico, no solo aplica para el concepto de belleza, sino para los demás datos recogidos y consiste en que diferencia el signo cuando este significa una cosa, como, objeto, espacio, persona $y$, cuando el signo no hace referencia a una cosa sino a un concepto como construcción cultural. De este modo, los signos identificables son, conceptos, cosas y sensibilidad:

Cuarto hallazgo del concepto de belleza también de tipo semiótico y que puede aplicar para algunos de los demás datos recogidos, consiste en que el signo denominado sensibilidad, puede contener un doble significado, uno, de tipo sensible y, otro, de función cognitiva superior. Ejemplo de esto son las expresiones, buenas energías, placer espiritual y demás.

Frente a la pregunta de lo sublime, los datos desde la sensibilidad y los conceptos son superlativos, exagerados y no presentan sorpresas, pero si constituye un hallazgo corroborado por Kant y que permite entender que lo sublime es una forma superlativa de percepción estética para estudiantes y profesores presentes en la muestra del Politécnico Jaime Isaza Cadavid y que permite entender sus percepciones estéticas. 
Hallazgos de lo agradable: El hallazgo frente al dato de lo agradable tiene aspecto de novedad y se encuentra en el aglutinante de la sensibilidad. De este, se infiere que lo agradable en los datos recogidos no solo contempla un interés sensible por poseer la cosa, a la manera de Kant, sino, además, hay una función cognitiva superior al asignar, cualidades de bondad a la sociedad, como significado de lo agradable o como, la de bondad en las personas. Esto, sugiere entonces, un hallazgo novedoso en términos de ampliación del significado por lo agradable. Respecto al de la belleza, contrario a lo esperado, y que va de la cosa a lo conceptual mediado por la sensibilidad.

Hallazgos de la estética: El hallazgo novedoso consiste en que, en la estética, el signo que responde la pregunta, no se refiere al juicio del gusto la manera que Kant la define, sino, a un conjunto de constructos culturales que se han hecho creencias y se manifiestan en los conceptos. En conclusión, se infiere que la pregunta por, qué es la estética, $\mathrm{y}$, según los datos, es la que más activa las funciones cognitivas superiores y la que menos requiere de la sensibilidad y las cosas a la hora de definirla. La mayor visibilidad de los constructos culturales frente a lo estético, no niega la presencia de la sensibilidad en relación a los fenómenos, sino que se invisibiliza en las respuestas.

Hallazgos con la pregunta por el amor: Como conclusión en la pregunta por el amor se tienen dos hallazgos. El primero es que los datos a pesar de tener una aparente equitativa distribución entre conceptos, personas y sensibilidad, las dos últimas tienen referentes que modulan los datos hacia los conceptos. Se ve como, ni las cualidades físicas de las personas, ni la sensibilidad por la sensibilidad cuentan para la definición de amor, y esto, demuestra un signo con un alto constructo socio cultural. El segundo hallazgo consiste en que la pregunta por el amor como un sentimiento que se siente por las personas tiene como consecuencia, una acción.

\section{Relaciones y asociaciones estéticas}

La sensibilidad en el dato aumenta y hace explicito el hecho de que la sensibilidad del entrevistado es la que soporta una pregunta por ese lugar bello. Es decir, esto, indica en muchos casos relaciones de correspondencia donde a un estímulo corresponde un cierto tipo de sensibilidad que lo aprueba.

\section{Ilustración acerca de los Lugares bellos}

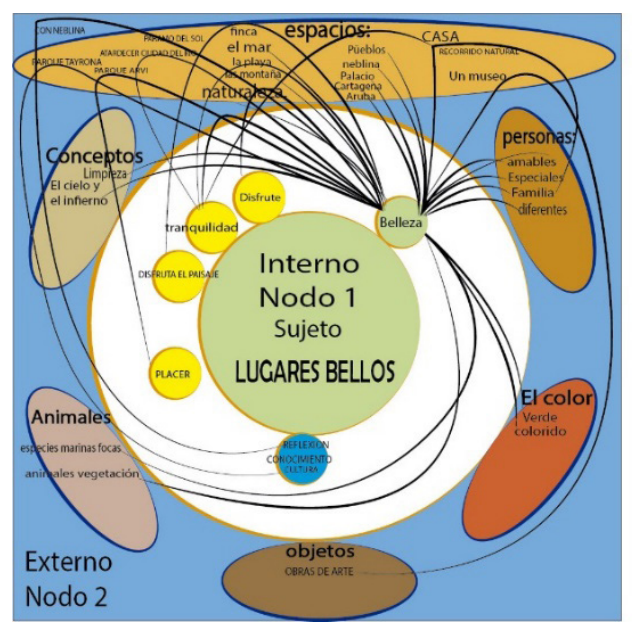

En las dos preguntas acerca de lo bello, es decir, en los conceptos como en las asociaciones de lugares, las funciones cognitivas superiores representadas 
en el círculo azul de los gráficos, que prende del sujeto, también aparecen como respuesta a un estímulo de belleza espacial. En los lugares bellos (ver gráfico anterior) como el Parque Tayrona y un segundo lugar, un espacio con neblina, las dos, estimulan las casillas en azul de conocimiento de la cultura y la reflexión, como una flecha doble que va a un espacio, y según las creencias de estas personas le generan conocimiento de la cultura al primero y una reflexión provocada por la neblina al segundo.

En la pregunta por los lugares bellos, la naturaleza es la palabra aglutinante que se lleva la gran proporción de las respuestas de los entrevistados. En una menor medida aparece la respuesta de la casa como espacio bello. Esto no constituye una novedad y de alguna forma estaba dentro de las expectativas. La explicación es que ya Kant ejemplifica los lugares naturales $\mathrm{y}$ funcionales como elementos que procuran placer estético, definidos como belleza libre y belleza adherente.

En tal sentido, Kant (1977) declara:

Hay dos especies de belleza; la belleza libre (pulchritudo vaga), y la simple belleza adherente (pulchritudo adherens). La primera no supone un concepto de lo que debe ser el objeto, pero la segunda supone tal concepto, y la perfección del objeto en su relación con este concepto. Aquella es la belleza (existente por sí misma) de tal o cual cosa; esta, suponiendo un concepto (siendo condicional), se atribuye a los objetos que se hallan sometidos al concepto de un fin particular. Mas la belleza de un hombre (y en la misma especie, la de una mujer, la de un niño), la belleza de un caballo, de un edificio (como una iglesia, un palacio, un arsenal, una casa de campo), suponen un concepto de fin que determina lo que debe ser la cosa, y, por consiguiente, un concepto de su perfección; esta no es más que una belleza adherente. (p. 61).

Hallazgos acerca de la descripción de un lugar bello: El principal hallazgo del dato, que además corrobora a Kant, consiste en que los lugares bellos estimulan las funciones cognitivas superiores, como lo demuestra el conocimiento de la cultura estimulado por la visita al parque Tayrona y la reflexión provocada por la neblina de un lugar. El segundo hallazgo del dato, Consiste en que los espacios naturales son la fuente que en su mayoría eligen los entrevistados para asociar la belleza de un espacio.

\section{Placer y emoción vivida}

En esta pregunta relacionada con el tema de los placeres y las emociones se les pidió a las personas que relataran, describieran una emoción vivida, agradable o desagradable que hayan tenido y que se les quedó en su memoria por su intensidad y persistencia. Aquí las 
respuestas presentan como reiterados los aglutinantes de personas y acciones. Los dos aglutinantes y sus referentes se diferenciaron deliberadamente por emociones bellas y sublimes, entendida la primera como agradable y la segunda como desagradable por el grado de violencia que conlleva la experiencia.

Respecto a la experiencia vivida se destaca la respuesta de un estudiante que responde que una emoción de rabia e injusticia producida por el castigo injusto de sus padres y que lo llevó a acabar con todo en su alcoba. Esto demuestra una vez más que donde se preguntaba por la definición del amor, la persona contesto que, es un sentimiento que me mueve a la felicidad, en este caso, la acción generada por el estímulo de rabia llevó al estudiante a actuar con violencia acabando con todo lo de su alcoba.

Primer hallazgo de una emoción vivida: Está ligado a un hallazgo anterior donde se pregunta por el amor y en los dos el estímulo genera una acción coherente con el tipo de estímulo. En el caso del amor, entendido por Kant como algo bello, el estímulo generó un movimiento hacia la felicidad; en el presente caso de la emoción vivida, la rabia por algo considerado injusto, entendido por el mismo Kant como sublime, llevo al sujeto a la destrucción de su alcoba. Esto puede concluirse en que los estímulos bellos conllevan reacciones bellas y los estímulos sublimes, reacciones sublimes.

Por otra parte, la pregunta acerca de las emociones estuvo marcada por las acciones y las personas como aglutinantes mayores, sin embargo, algunas de estas acciones muestran la adquisición de objetos como cámara, computador y apartamento una tendencia fuerte que liga las acciones a los objetos. Kant, definiría esta experiencia como lo agradable, dado que lo agradable dista de lo bello en cuanto que lo primero tiene un fin de uso y su experiencia estética se agota cuando se agota la experiencia de su uso o consumo, mientras por otra parte lo bello o sublime no se agota en cuanto ambas experiencias no tienen un interés de uso, están más cerca de un ejercicio de la imaginación y el intelecto como ya se ha expuesto anteriormente.

\section{Ilustración acerca de una Emoción vivida}

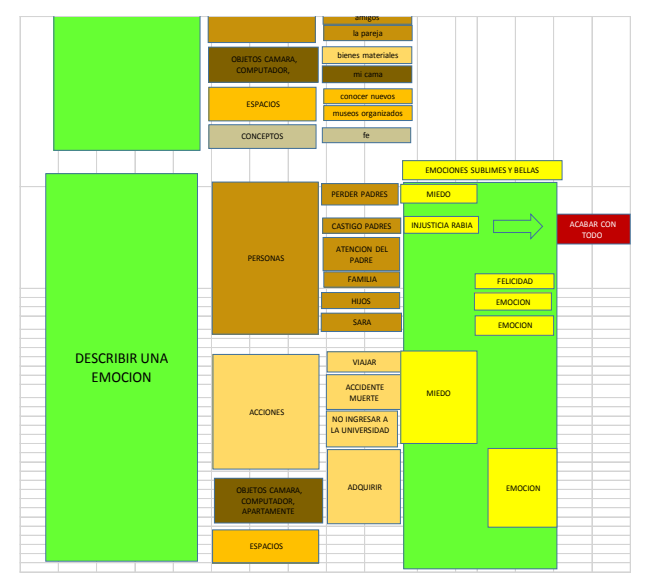

De nuevo está el espacio de paisajes como otro de los elementos que una persona recuerda que le genera placer. Ya en la primera parte de conceptualización, el paisaje natural apareció como aquel que las personas elijen como espacio de belleza y placer, pero aquí el dato tiene una complejidad mayor, porque este paisaje está ligado a un conjunto de estímulos de acciones 
y reacciones que el entrevistado la relaciona en la construcción de su repuesta.

Esto es lo que dice el docente, Aguirre (2018)

Recientemente viajé a Bogotá con mi hijo Tomás y nos detuvimos en un punto en la carretera (Alto del Cocora) era temprano en la mañana y el sol reflejaba de manera muy bella el paisaje del valle que se abría a nuestra vista; por un momento compartimos la visión de una belleza extraordinaria que nos emocionó mucho (entrevista).

El tercer hallazgo de placer y emoción vivida: Consiste en que dichas experiencias no se definen en un solo aglutinante, sino que reúne un conjunto de aglutinantes como, tiempo, personas y amor en un espacio y tiempo determinado.

\section{Asociaciones del Placer}

Luego de las descripciones de placeres o emociones vividas se les pidió a las personas que respondieran ¿Con qué relaciona la palabra placer? las acciones y posteriormente las personas fueron los mayores aglutinantes. Es decir, las acciones son un aglutinante que van acompañados de personas, y viceversa, cuando el aglutinante son la personas, sus referentes conllevan acciones.

Primer hallazgo de las asociaciones de placer: Es similar este hallazgo al tercero de la pregunta por emociones vividas. Este consiste en que las asociaciones están compuestas de más de dos aglutinantes en simultáneo.

Si se observan las acciones que generan placer en las personas entrevistadas hay un elemento común y es que todas tienen de por medio uno de los cinco sentidos. Las acciones que involucran los sentidos son, comer, desayunar, oír música, tener sexo, ver algo, ver imágenes, viajar, observar y ser observado, en todas, la mediación de los sentidos con el estímulo es obligada; lo que se quiere decir y se explicará a continuación por contradicción o ironía, es que las respuestas no relacionan el placer de las acciones con las funciones cognitivas superiores, por ejemplo, nadie dice que le procura placer cuestionar el desayuno, pensar la música, abstraer el sexo, o argumentar un viaje, estas funciones cognitivas superiores como prefijo de una acción no concuerdan, no dicen nada lógico. Lo que, si le procura placer al sujeto entrevistado, es, sentir las acciones.

Segundo hallazgo acerca de las asociaciones del placer: Este hallazgo en las asociaciones del placer que hicieron los entrevistados tiene que ver con la relevancia de la sensibilidad como referente modulador del aglutinante, acción. Los sentidos, son los receptores de la acción placentera. 
Ilustración acerca de las Relaciones de placer

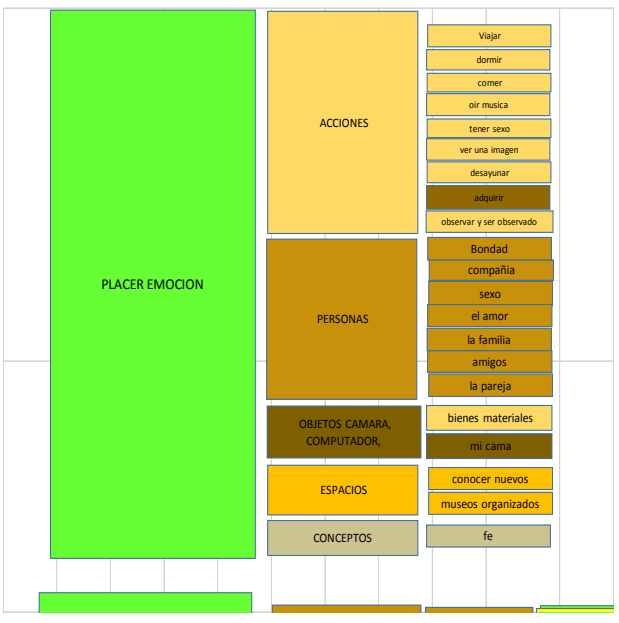

Tercer hallazgo en las asociaciones del placer: En este hallazgo, acerca de lo que las personas asocian con placer, los sentidos les permitieron percibir las acciones placenteras, mientras que, la sensibilidad por los sentimientos, les permitieron valorar el placer que le procura una persona. Consecuente con lo anterior, el dato se comporta también de modo diferente, es decir, en los sentidos, el objeto es acción, en los sentimientos, el objeto está en el producto de constructos intangibles de la cultura, llamado, sentimientos, como, el amor o la bondad. Estos, son signos que nacen de otros signos y de otros más y que tienen a las personas, sólo, como referentes físicos. No se percibe, se entiende.

Como ya se dijo, Kant, plantea que finalmente es en el sujeto quien radica la experiencia estética, más que en el objeto, en tanto que algo puede ser placentero para una persona para otra no; así mismo, Peirce lo dice de otro modo "...es la creencia, que habita al sujeto, la que genera las acciones" en consecuencia, un mismo estímulo puede significar para una persona el inicio de una acción mientras para otra simplemente no es estímulo a ninguna acción.

Cuarto hallazgo acerca de las asociaciones de placer de las personas: Este hallazgo está precisamente, en el hecho, de que, el placer busca el placer cuando este se pierde. Las creencias llevan a realizar acciones, que, a su vez, les renueva el placer. Es decir, un proceso de estímulo, creencia, acción, que, puede iniciarse indiferentemente desde el estímulo o desde la persona. La reflexión acerca de lo que está primero nos llevaría al origen de nuestra existencia y de la cultura misma. Lo que sí es evidente, es que el sujeto, el objeto y el signo dejan de verse como elementos separados para ser uno en su interacción, deja la dualidad de sujeto, objeto y ahora, el signo, crea la una terceridad (Peirce).

Ilustración acerca de la Relación de placer y academia

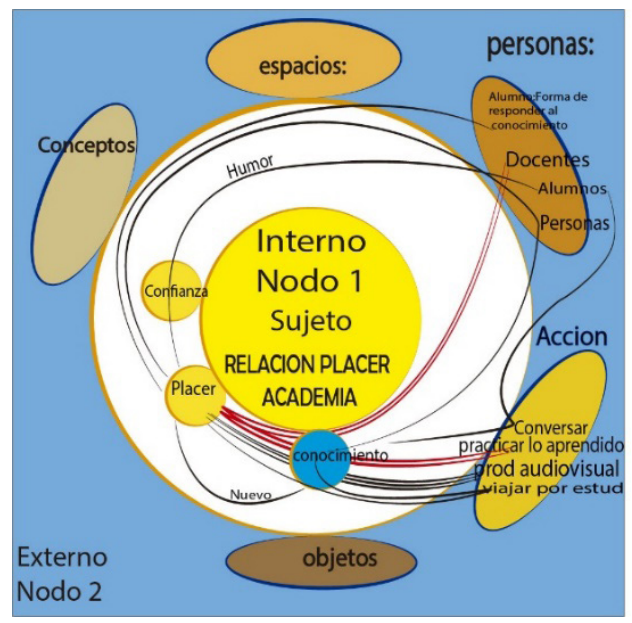


Un estudiante (2018) agrega "yo digo que para mí es muy placentero cuando te imparten unos conocimientos y luego le pide que ponga en práctica sus conocimientos es muy placentero cuando tú lo logras sientes que tuve ese conocimiento que te dieron te sirvió".

Segundo hallazgo en la relación entre personas y placer por el estudio es que las personas y las acciones representan los dos únicos aglutinantes en la asociación que los datos establecen entre el estudio y el placer, mas, de las personas, sólo aquellas que son inteligentes y pueden comunicar lo que saben, son generadoras de placer en el estudio.

Los datos tienen reiteraciones en los estudiantes cuando expresan que el conocimiento es placer, mas, este conocimiento no es cualquier tipo de conocimiento, pues éste, está modulado por referentes, uno de ellos es el interés frente a lo nuevo y el otro por la práctica del objeto de conocimiento que finalmente también se traduce en novedad o transformación de la monotonía del salón de clase.

Tercer hallazgo en la relación del placer con el estudio se refiere a la cualidad que tiene la acción siempre de ser novedosa. Placer en los nuevos conocimientos, en la práctica por oposición a la teoría, en la predilección de nuevos espacios y demás.

Cuarto hallazgo que relaciona el placer con el estudio, este hallazgo surge de la relación entre el primero y el segundo hallazgo. Este consiste en que el placer que sienten por las personas que saben comunicar sus conocimientos contiene una novedad, esta novedad, es el nuevo conocimiento.

Quinto hallazgo en la relación placer con el estudio: Este hallazgo es de tipo semiótico y se desprende de los anteriores. El signo, en ninguno de los tres anteriores hallazgos se refiere a una, cosa, como significado, ni el conocimiento, ni la capacidad comunicativa, ni la novedad. Esto hace de este hallazgo uno de los de más alto constructo sociocultural analizado hasta el momento, el cual, además, exige de un gran ejercicio de la imaginación para poder entender sus significados.

\section{Aquello que más admira en compañeros y docentes}

En la pregunta por el placer ha aparecido una y otra vez el entendimiento. Entender por medio de la imaginación es fuente de placer, dice Kant. Esta fue la pregunta que se hizo. ¿Qué es lo que más admira de sus compañeros y docentes? y este placer intelectual, muy de moda en los círculos artísticos y cultos de la sociedad presentó los siguientes datos. Lo que más se admira entre docentes y estudiantes juntos son cualidades o referentes en las personas que tiene que ver con su capacidad intelectual, de entendimiento y de resolver situaciones que dependen de las capacidades cognitivas superiores.

Más allá de bondad o cualidades éticas de una persona que solo aparece en unas pocas respuestas, las funciones cognitivas superiores están presentes en expresiones como, capacidad de resolver, hacer sencillo lo complejo, 
capacidad de cuestionar, apertura mental, capacidad de transmitir el conocimiento, humor, curiosidad, frente a conceptos de tipo ético como, capacidad de compartir, honestidad, bondad, valorar la clasey ganas muestra la dominancia de las respuestas hacia la inteligencia como aglutinante principal en la pregunta por la admiración. Es claro entonces afirmar que para las personas preguntadas la admiración que profesan por el entendimiento $e$ inteligencia está por encima de sus cualidades éticas.

\section{Belleza, bondad e inteligencia}

En esta ocasión al poner a elegir a los entrevistados entre tres opciones explicitas, la inteligencia cedió espacio frente a la bondad y esta se creció, ocupando prácticamente el mismo nivel y valor que la inteligencia. Es también de anotar que la belleza desapareció en las respuestas. En la inteligencia se aglutinan la mitad aproximadamente de las respuestas acerca de esta triple elección brindada para elegir y en la bondad, la otra mitad.

Los referentes de este aglutinante de inteligencia son,

la creatividad, expresadas de modo en que se pueden contar cosas, como que, la inteligencia crea una mentalidad abierta; mientras que el aglutinante de la bondad, similar al anterior por cantidad de respuestas, admira en la bondad, el agradecimiento, la amistad con los compañeros, la capacidad de ayudar.
Ilustración acerca de belleza, bondad o inteligencia

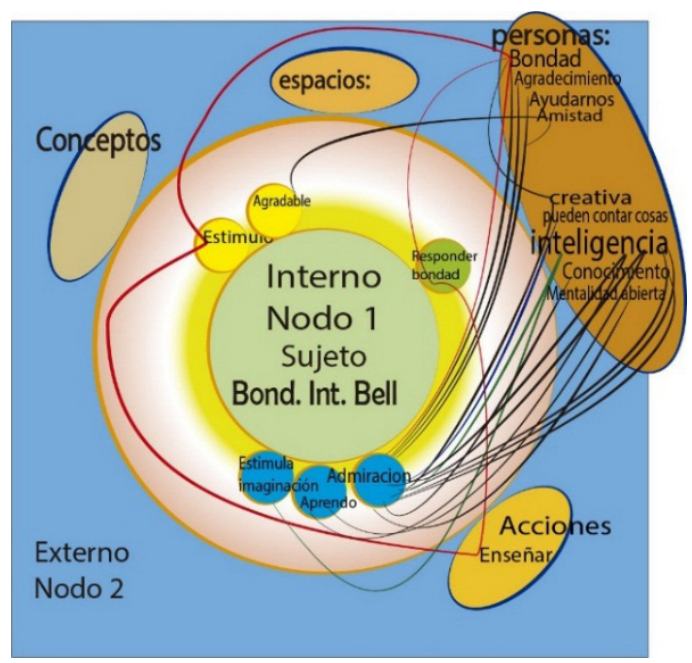

Lo más destacado en las respuestas a esta pregunta de elección triple radica en que, como lo muestra el grafico superior, la línea roja que va y vuelve de sujeto a objeto en seis ocasiones, representa la respuesta de un docente, que, continuando con el elemento de acción reacción expuesto en párrafos anteriores, aquí, el dato, contiene un anticipo del sujeto, docente, que busca, a partir de un tipo de estímulo bondadoso de su estudiante, generar el mismo trato bondadoso con su enseñanza y así crear una acción y reacción continua. Este es textualmente el dato.

\section{Respuesta de un docente (2018)}

Pensaría que la bondad de alguien en el salón es un estímulo mayor para ir a enseñar, porque me genera mayor compromiso personal de corresponder su bondad con mi tarea de enseñar. 
El primer hallazgo en relación con la pregunta tripartita acerca de la admiración, consiste, en que continuando con algo ya hallado en preguntas anteriores, el dato, acerca de la admiración por la bondad, contiene tres pares de acciones reacciones que dibujan dinámicas de causas y efectos que permiten al sujeto, prever en otro sujeto, las consecuencias de sus acciones.

La misma triada que pone la belleza, la bondad y la inteligencia como estímulo para lo académico presenta un dato a analizar de un estudiante, el cual, manifiesta que la inteligencia de un profesor es la que más la ha motivado $\mathrm{a}$ ir a estudiar porque dicha inteligencia le estimula la imaginación. Aquí es imposible analizar el dato sin que se asemeje a Kant. En él, la imaginación es estimulada por la belleza y aquí por la inteligencia. En Kant la imaginación ante lo bello busca el entender.

\section{Imaginación y creatividad}

La primera pregunta para analizar es ¿Qué concepto tiene sobre creatividad? $\mathrm{y}$ es con respuestas aglutinadas en conceptos $y$ en segundo término con acciones que mayoritariamente respondieron. Se presentan las dos al tiempo, porque los conceptos y las acciones están relacionadas entre sí en los datos como referente que modula el aglutinante. Los verbos, construcción, solución, imaginar, inventar, proponer fueron los referentes que modularon este aglutinante de los conceptos. Sin embargo, si se les observa, los conceptos y las acciones están descritas en cada palabra de forma simultánea, es decir, el significado de cada dato fluctúa entre una función cognitiva superior y una acción física.

De nuevo aparece lo nuevo, no es una cacofonía. Los datos acerca de la definición de creatividad muestran cuatro respuestas donde las acciones son el aglutinante y el referente es, nuevo, las nuevas maneras de hacer algo, es el deseo de hacer cosas nuevas $y$ hacer algo nuevo son referentes que modulan este aglutinante de acción. El término nuevo y acción comienzan a ubicarse como referentes reiterados en la respuesta por la creatividad, en este caso, y del placer por el estudio en preguntas anteriores.

\section{Ilustración acerca del Concepto de creatividad}

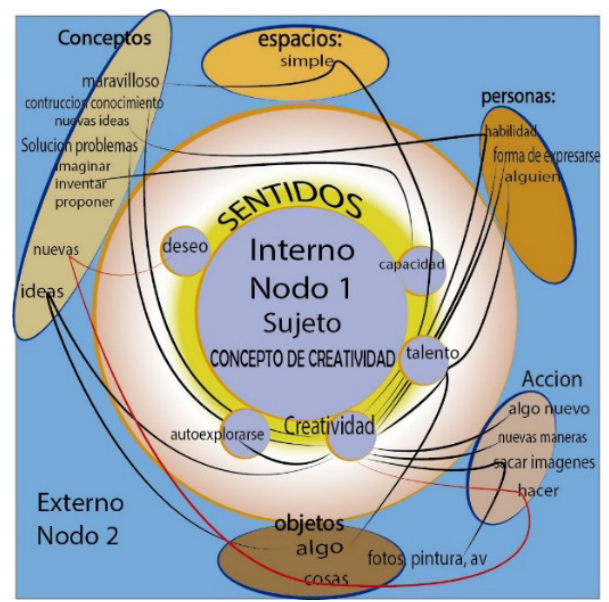

Otro elemento que se destaca en la definición de creatividad son las cualidades de las personas. Cualidades como, forma de expresarse, talento, habilidad para generar nuevas ideas, capacidad, deseo de hacer nuevas cosas, imaginar, inventar y proponer son todo el abanico de significados para 
la palabra creatividad aglutinado desde las personas. Estas cualidades, que son también formas de acción creativa, están ligadas a las diferentes formas de reacción propias de cada persona frente a un mismo estímulo, y en esto, volvemos al mismo punto de Kant y de Peirce ya formulado anteriormente, donde lo que nos diferencia como sujetos está en las reacciones propias de cada individuo frente a un mismo estímulo.

El segundo hallazgo en la pregunta por lo que es la creatividad consiste en que el significado de una persona creativa radica en su capacidad de generar novedad. En este sentido la definición de que alguien es o no creativo depende de su propia reacción frente a los estímulos, si esta reacción es convencional, tradicional, esperada o conocida la persona no es considerada como creativa, si por el contrario, la reacción es inesperada, nueva, novedosa $\mathrm{y}$ finalmente inaugura una manera poco conocida o desconocida hasta ahora, se considera, que es una persona creativa.

Tercer hallazgo en la pregunta por la creatividad: Este tercer hallazgo consiste en relacionar las respuestas entre creatividad y placer por estudiar. La creatividad es novedad en la acción y en las cualidades intelectuales de las personas; el placer por el estudio es, novedad y acción. En esto consiste este hallazgo, en la reiteración del aglutinante acción y novedad en dos preguntas diferentes de creatividad y placer por el estudio.

\section{Acto creativo}

El primer hallazgo en la pregunta por un acto creativo: Este primer hallazgo en la pregunta por describir un acto creativo consiste en relacionar el aglutinante de sensibilidad para describir simultáneamente un acto creativo, así como lo es de definir la belleza, lo agradable y el amor.

El segundo hallazgo en la pregunta por el acto creativo consiste en ir más allá de relacionar conceptos de belleza, con el acto creativo. Es decir, que además de responder a la pregunta por la belleza, lo agradable, el amor y el acto creativo con una gama de sensaciones y sensibilidad. Esta sensibilidad, tiene un doble componente de significado, uno, de tipo sensible y, otro, de función cognitiva superior. Ejemplo de esto son las expresiones, me siento como un niño, es un temor placentero, siento mariposas.

\section{Ilustración acerca del Acto creativo}

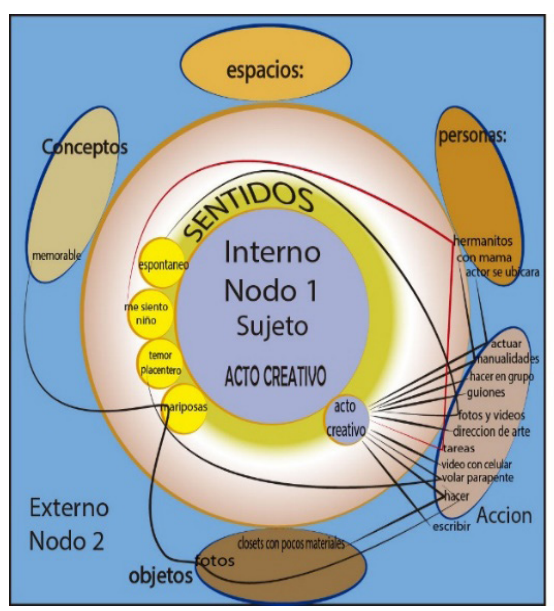


La importancia de la sensación de placer que siente el entrevistado al describir su acto creativo es que este es un motor, que, de forma figurada, inicia la construcción de un siguiente acto creativo para poder suscitar en el sujeto la sensación de placer que perdió, esto, en una dinámica que entre ausencia y presencia de placer mantiene al ser humano en búsqueda permanente de hacer presente el placer ausente. Así el tercer hallazgo acerca del acto creativo consiste en que el placer sentido en el acto creativo se convierte en un motivo que se busca cuando se pierde.

El cuarto hallazgo del acto creativo: Este hallazgo consiste en relacionar, la acción creativa en búsqueda de placer, con, la intención de agradar a otros para que ese otro procure agradarme a mí. En una acción circular de presencia ausencia, causa efecto. Placer que se auto regenera permanentemente en la acción creativa ética y estética.

\section{Estimular la imaginación}

¿Cómo las personas entrevistadas estimularían la imaginación en alguien? las respuestas encontraron un gran aglutinante de las acciones. En el gráfico inferior, se pueden ver los referentes que modulan el aglutinante de la acción como forma efectiva de estimular la creatividad tienen un elemento común a pesar de lo disimiles que parecen.

\section{Ilustración acerca de Estimular la imaginación.}

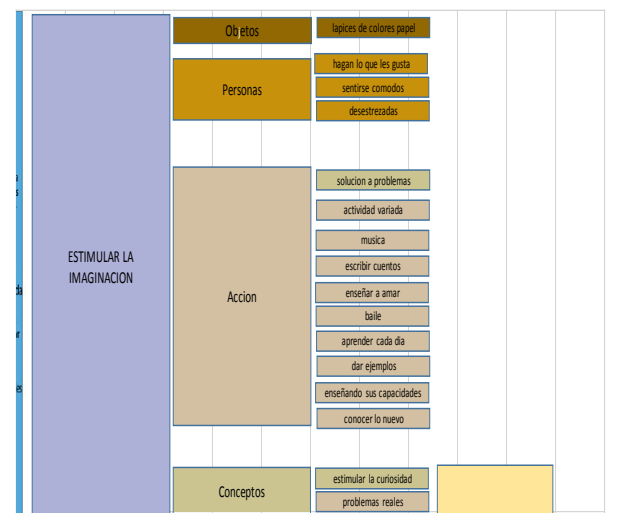

Otro elemento que se reitera es un referente tanto de los aglutinantes de acción como de personas y tiene que ver con el placer expresado de diversas formas, hagan lo que le gusta, sentirse cómodos, des estresados, escuchar música, bailar estas formas variadas de placer y distensión que describen un estado necesario, según los datos, para la creación. Así, el primer hallazgo acerca del estímulo a la imaginación consiste en que las formas variadas de distención y pacer, según los datos, estimula la imaginación.

En este estímulo algunas respuestas estimulan sin acción y otras con acción. Es decir, en expresiones como sentirse cómodas, conocer lo nuevo o des estresadas en este caso se habla de estimular la imaginación, que no es igual pero si similar a la creación, cuando dice, escribir cuentos o actividades variadas porque, como hermanas, tienen en común que las dos tienden un lazo que las une, lo nuevo; la imaginación crea lo nuevo en el entendimiento que no se tenía antes sobre algo, la in acción, y, su hermana, 
la creación, que encuentra la novedad del demiurgo que crea materializando la imaginación

El segundo Hallazgo acerca del estímulo de la imaginación, aquí, se establece una diferencia al tiempo que una unidad entre el estímulo de la imaginación y la creatividad. El primer estímulo se presenta en la inacción mientras que el segundo en la acción. Así mismo, los dos se presentan unidos frente a la novedad, el primero por conocer algo nuevo el segundo por crear cosas novedosas.

\section{Aulas que estimulan la imaginación}

La primera pregunta formulada fue ¿Cómo sería para usted un aula de clase que estimula la imaginación? Ante esta pregunta se puede identificar de mayor a menor la cantidad de respuestas de los entrevistados, este orden es: cualidades físicas del aula, el color y la luz (se convierte en un aglutinante debido a la reiteración y las referentes que lo modifican), las personas y los objetos en una tercera posición y finalmente las acciones.

$\mathrm{Si}$ bien es cierto que las cualidades del espacio ocupan el primer lugar, estas cualidades están referenciadas por moduladores que remiten a otros conceptos que no es el espacio mismo. Por ejemplo, de los seis referentes tres de los datos se responden desde la sensación de placer que pueden producir en los entrevistados, por ejemplo: Las aulas abiertas permiten ver el mundo, dos, aulas con distribución igualitaria permiten la comunicación, tres, aulas en otros lugares diferentes al aula de clase tradicional otorgan más motivación; las tres, se soportan y responden en las sensaciones del sujeto, las cuales, se pueden resumir en sensaciones de placer. Aquí, entonces, el placer, en palabras como, libertad, relajamiento, comunicación y variedad resumen los referentes que modulan el aglutinante y que son las características que debe de tener, para los entrevistados, las aulas que estimulan la creatividad.

\section{Ilustración acerca de las aulas que estimulan} la imaginación.

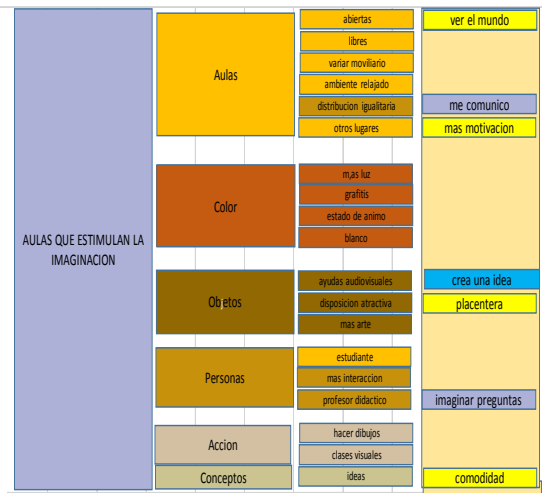

El color finalmente se convierte en una especie de aglutinante por efectos de la reiteración y sus pertinentes referentes que lo modulan, pero, si se observa, nace como un referente del espacio. Más luz, más grafitis, colores de acuerdo a los estados de ánimo y el blanco son los referentes de este aglutinante. Entre sí, ellos, no tienen nada en común excepto que el blanco emana mayor cantidad de luz y los dos, dejan claro, la claridad que debe de tener el espacio para estimular creatividad; los grafitis, sugieren la libertad de expresión y este se puede llegar a relacionar con el estado de ánimo expresado por otro entrevistado, sugiriendo, que la expresión de las 
emociones y las ideas hacen parte de las condiciones de los espacios que estimulan la creatividad.

Los objetos del aula creativa tienen preferencias como estimuladores de la creatividad en dos sentidos, uno, por las ayudas didácticas, porque estas permiten crear una idea de lo que se habla, y dos, está la preferencia por una disposición atractiva de los objetos en el salón de clase porque de este modo se genera placer. La creencia aquí radica en que ver lo que se habla en una pantalla y el placer de una atractiva disposición de los objetos tiene la capacidad de estimular la creatividad. De nuevo, el placer y las sensaciones del sujeto son los criterios, según el dato, para la aprobación de que un espacio estimula la creatividad o no.

En las aulas que estimulan la creatividad, las ayudas didácticas tienen en sí mismas la función de lo estético, similar a la sensación de atractiva disposición de los objetos en el aula. Esto, se explica porque la imagen icónica de la ayuda didáctica que es la pantalla de video o imagen fija es un signo que no requiere conversión de su significado, esto, porque se parece a la cosa que representa; diferente, a la palabra como símbolo, que al no parecerse a la cosa, si requiere un esfuerzo del estudiante para abstraer su significado desde el lenguaje verbal y convertirlo en imagen mental. La imagen de la pantalla es entonces algo más fácil de entender y por ende más placentero y estético que la palabra simbólica, la cual requiere transformarla mentalmente en imagen y poder entender su significado.
Otro hallazgo en términos consiste en que la distribución igualitaria entre docente y estudiante propicia la comunicación. Esto lo dice un profesor, que describe que las distribuciones en mesas redondas en vez de la disposición de sillas de la forma tradicional del aula de clase le han permitido asumir la clase con una mayor fluidez de la comunicación con sus estudiantes.

Finalmente, cuando el profesor es didáctico le permite al estudiante imaginar preguntas. En esta respuesta subyacen dos elementos a destacar, el primero es que didáctica en este contexto sugiere actividad, movimiento, no solo físico, sino, sobre todo, mental; el segundo, es que esta actividad mental no es otra cosa que estímulo a la imaginación. Aquí, movimiento, novedad, imaginación y didáctica se mueven en el mismo sentido para que la persona facilite con libertad el entendimiento del concepto que desea comunicar el docente. Esto, se ha visto anteriormente en otras respuestas que involucran acción y novedad como placer en el estudio.

\section{Espacios creativos}

Coherente y siguiendo la misma línea de la pregunta anterior se indago a las personas acerca de ¿cómo es un espacio creativo? Aquí las respuestas se limitaron en general a describir las características de este espacio, pero con unas características que dependen en gran medida de los sentidos, sobre todo, de la vista y el oído. Palabras como: estimulante, sinestésico, mesa redonda para mirarnos, poder acostarse en el suelo, salones abiertos y redondos, 
música moderada, luz natural, buena luz, poco ruido, salón blanco, vacio, diferentes ambientes son todas estas cualidades que están mediadas por los sentidos de la vista, el oído y en menor medida por el tacto. Los sentidos entonces son la forma en que las personas prefieren usar para definir las características de un espacio creativo.

\section{Tesis}

En el inicio de este proyecto de investigación estuvo la inquietud acerca de la separación percibida entre el placer con que se vive el tiempo libre y el deber con se asume el estudio y la sensación de que esto no debe de ser de este modo. Así, por el contrario, el placer por aprender debía de ser su sentido fundador. De este momento a hoy ha pasado ya casi dos años y esta pregunta va encontrando respuestas. Sin embargo, esta tesis ha salido de la estética y el placer en el aprendizaje universitario hacia la vida. Esto, porque las respuestas tocaron temas que van más allá de la percepción del espacio y las relaciones cotidianas universitarias hacia la pregunta por el placer que nutre la existencia de las personas.

Kant como referente teórico de este proyecto fue el primero en abrir las inquietudes por la estética a una gama bastante amplia de temas, pero todo, gira alrededor del, placer. El placer ante lo estético, lo ético y el conocimiento abrió las puertas de esta investigación. Posteriormente, Kant, quedó corto a la hora de entender e interpretar lo que las informaciones y los datos arrojaron y Peirce dio método. Entender toda esta información solo era posible si se tenía un método. La visión triádica de este autor permitió entender y darle sentido al gráfico que ilustra cada una de las respuestas dadas en una relación dinámica de tres elementos, interpretante, signo y objeto.

Ya entrados en tema las siguientes constituyen las tesis más claras de la presente investigación. Se presentarán en un orden similar al realizado en las preguntas y los objetivos, sin embargo, algunas respuestas del inicio hallaron conexión con otras del medio y el final brindando una visión de mayor simultaneidad de las tesis a las cuales se llegaron y menos de causa efecto. Esto redunda en coherencia con el método de interpretación seguida en la recolección e interpretación de los datos que no es binaria si no triádica y simultánea.

La belleza presenta dos grandes versiones, una, la cultura clásica griega donde lo visual es manifestación del mundo proporcionado que otorga el número, $\mathrm{y}$, cristiana, como alusión al interior de las personas, de forma más precisa, su bondad. En cuanto a lo que refiere al interpretante, él, en ocasiones, define la belleza y la sublimidad desde la emoción que les hace sentir y en otras ocasiones con la razón, que intenta entenderla. Lo sublime, presenta siempre un grado alto, superlativo. Lo agradable, una tendencia casi exclusivamente hacia la emoción. En todo lo anterior no se presenta un hallazgo a manera de novedad con la teoría estética de Kant.

Lo que se refiere al concepto de la estética las definiciones o signos usados, estos, la presentan desde un 
área técnica, especializada que trata de entender y reproducir la imagen de una cierta manera que para ningún caso se refiere a lo bello, lo agradable o lo sublime de un signo, como si sucede en Kant. Esto, marca la novedad respecto a la estética. La estética no hace juicio frente a lo bello o agradable como en Kant, es más, una técnica del signo para comunicar y expresar.

El amor está ligado a la acción, es, a la manera de Peirce, lo energético del interpretante frente a la experiencia de recibir y producir signos, en este caso, referidos al amor. Este energético amoroso está ligado a la lógica y la razón por medio de la emoción. La reacción triádica, es una acción de entendimiento, de asimilación de constructos culturales que se hacen creencia y acción energética del interpretante.

Los lugares naturales considerados bellos estimulan el entendimiento, la emoción y el energético del interpretante, porque, aunque las creencias, los constructos y las acciones finalmente dependen del sujeto (interpretante), se recopiló evidencia, que en términos generales, se puede predecir que los estímulos emocionales bellos conllevan reacciones bellas y los estímulos sublimes a reacciones sublimes. Las primeras acompañadas de emociones moderadas y agradables, las segundas acompañadas de emociones superlativas, exageradas y en ocasiones consideradas como desagradables.

El placer está en la percepción sensible $y$ en el entendimiento racional. En el primer caso la percepción sensible de las acciones y las cosas; por otra, en entender los sentimientos propios o de otra persona. En todos se siente placer y amor. En la percepción se puede sentir placer producto de un objeto tangible como las cosas o la acción, o, por otra parte, se entiende desde lo intangible como la bondad y los sentimientos de una persona. El primero se percibe por los sentidos mientras que el segundo por acción de la lógica y la razón creadoras de las creencias que elabora el interpretante. Es decir, el placer no se percibe, se entiende.

La acción no sólo va ligada al amor y el placer en general sino además frente a las asociaciones del placer con el estudio. Aquí, las acciones y las personas continúan siendo las fuentes del placer. En las personas, solo aquellas que de forma inteligente logran comunicar lo que saben. Mientras que las acciones están moduladas por la novedad, por ejemplo, de los nuevos espacios, de los nuevos conocimientos. $\mathrm{Si}$ se hace una intersección entre personas que saben comunicar lo que saben y acciones novedosas, la tesis, es que las personas que generan acción física o de la imaginación y que tienen algo nuevo que comunicar son fuente de placer académico.

El placer por el estudio no depende de una cosa u objeto, sino y según las respuestas anteriores de exclusivos constructos culturales como lo es la concepción de novedad y capacidad comunicativa. Esto, hace que las cosas no estimulan, según los datos, el placer en el estudio. Esto, puede tener dos explicaciones. Una, que efectivamente no sean necesarios en el estímulo por 
el estudio, dos, que esta respuesta puede evidenciar que la carencia de los mismos en el espacio de aprendizaje y la escaza experiencia de las personas con los estímulos visuales ha llevado a las personas a no tenerlos presentes. Lo anterior se demostrará en párrafos posteriores que le segundo caso es el correcto.

Respecto a la admiración se puede afirmar que al igual que la bondad y los sentimientos de una persona, estos no se perciben por la emoción sino por la lógica y la razón. La admiración por las personas está dada primero por la inteligencia, la admiración por la bondad existe cuando se nombra como opción de elección. Espontáneamente se elige a la inteligencia. Lo que denota un auto de corrección moral para no ser juzgado como frívolo. Esto no es una afirmación hecha sin evidencia. Como se vio en párrafos anteriores, las reacciones de las emociones están mediadas por la activación de la razón que planea con las propias acciones agradar o molestar al otro y predecir la reacción del otro.

La creatividad se define como la acción energética novedosa del interpretante y en este sentido la definición de que alguien es o no creativo depende de su propia reacción energética frente al signo. Si esta reacción es convencional, tradicional, esperada o conocida la persona no es considerada como creativa, si por el contrario, la reacción es inesperada, nueva, novedosa o desconocida inaugura lo nuevo o escaso y se considera que es una persona creativa. La sensibilidad y el entendimiento se unen para percibir la novedad, no tienen códigos que mecanicen esta percepción y por tanto deben de construirlos, imaginarlos o crearlos. Se toma el máximo de atención para esta acción.

La novedad presente en el acto creativo es fuente de placer, tal, como se afirmó en párrafos anteriores. Este placer se posee y no se posee de forma alternada, generando más que un círculo vicioso una fuente de energía que se auto procura el placer perdido de forma reiterada y permanente. De este modo, la acción anticipada que se vio en párrafos anteriores constituye además una de las formas que tiene el sujeto de auto procurarse placer, y es, procurar placer a los demás.

El placer y distención están en la base de todo estímulo a la novedad cognoscitiva o de acción. Esta novedad cognoscitiva o de acción puede ser de tres formas, la primera desde la inacción externa pero si interna del interpretante, llamada, imaginación, donde está presente el entendimiento y la emoción; la segunda, se da desde la acción energética que se desprende del interpretante, se consigna en algo novedoso y se llama creatividad, y la tercera, como la simultaneidad de las dos anteriores. Imaginación y creatividad presentes.

Continuando con la carencia de cosas a la hora de estimular la imaginación expuesta en párrafos anteriores y que se puso en duda dado que la falta de presencia y diseño de espacios y cosas en las aulas actuales reales hayan sido la causa de su no nombramiento. En el aula creativa se concibe que el espacio 
y sus cosas son la principal fuente de estímulo a la imaginación. Los espacios naturales, con luz, sin muros, que presenten flujo visual desde donde se sienta y vea el mundo, mesas redondas de relaciones horizontales entre estudiante y docente son aulas que estimulan la imaginación, el placer, el entendimiento, la libertad y la comunicación. Esto, demuestra entonces, que la carencia de espacios y cosas estimulantes para la imaginación no solo ha desestimulado la imaginación, sino, que ha construido la creencia de que no se necesitan. Esto, va en contra vía de lo que idealmente es y se considera creativo. Esto, no es otra cosa que el olvido de un valor estético, consecuencia del el uso y la costumbre de un antivalor estético. Es decir, un espacio anestésico ha construido creencias de aprendizaje anestésicas.

Respecto a la estética en las ayudas didácticas como videos y proyecciones, estas, tienen en sí mismas la función de lo estético. Esto se explica, porque la imagen icónica de la ayuda didáctica que aparece en la pantalla de video o imagen fija es un signo que no requiere conversión de su significado, esto, porque se parece a la cosa que representa; diferente, a la palabra como símbolo, que al no parecerse a la cosa, si requiere un esfuerzo del estudiante para abstraer su significado desde el lenguaje verbal y convertirlo en imagen mental. La imagen icónica de la pantalla es entonces algo que estimula el entendimiento y por ende es más placentero y estético que la palabra simbólica, la cual requiere transformarla mentalmente en imagen y poder entender su significado. Así mismo, la capacidad de comunicación del conocimiento de las ayudas didácticas está íntimamente relacionada con la capacidad de comunicación del profesor dado que las dos permiten hacerse una imagen mental para entender el conocimiento y las dos hacen parte de una forma de placer estético académico.

Finalmente, cuando el profesor es didáctico le permite al estudiante imaginar preguntas. Aquí subyacen dos elementos a destacar, el primero es que didáctica en este contexto sugiere actividad, movimiento, no solo físico, sino, sobre todo, mental; el segundo, es que esta actividad mental no es otra cosa que estímulo a la imaginación. Aquí, movimiento, novedad, imaginación y didáctica se mueven en el mismo sentido para que la persona facilite con libertad el entendimiento del concepto que desea comunicar el docente. Esto, se ha visto anteriormente en otras respuestas que involucran acción y novedad como placer en el estudio.

Como síntesis de todo lo expuesto, y haciendo una comparación entre todas las respuestas analizadas, tomo la idea semiótica de Peirce, sobre la relación del signo con el interpretante y el objeto como uno de los elementos de su semiótica, esta es la teoría.

El signo como mediación entre el objeto y el interpretante. Son los LENTES que median entre el objeto y el interpretante. Entiéndase objeto en su sentido amplio, cualquier Phaneron (fenómeno). A su vez Peirce, dice que se da en tres posibilidades, que se pueden distinguir pero no disociar: 
El lógico-racional, media entre el emocional y el energético, por lo general nos quedamos en los dos de abajo y no avanzamos al lógico racional, pero este siempre está en la posibilidad de ejercitarse. El energético, siempre es una acción de algo sobre algo.

\section{Conclusión}

La inquietud inicial acerca del exceso de deber y poco placer percibido en el proceso de aprendizaje universitario actual es real. Esto se deduce, de que ni las cosas que contienen los espacios de aprendizaje, ni los espacios mismos, ni las acciones constituyen los ideales de aquello que las personas conciben como placenteros. A pesar de esto, las personas, constituyen los mayores generadores de placer.

Lo anterior se desprende de la carencia de elementos estéticos en las aulas de clase normales de la actualidad que estimulen el placer estético de las personas, el diseño de salones encerrados, lejano a los diseños de salones abiertos, con presencia de la naturaleza, la luz y el color; con sillas y disposiciones espaciales que escapan a toda comunicación igualitaria $y$ que favorece las relaciones de poder por parte del docente y en general lejano de todo lo que las personas consideran placentero; las acciones limitadas, repetitivas y mecanicistas en las dinámicas de clase, lejanas de todo lo novedoso, práctico y que las personas consideran estimulante a la imaginación.

La ausencia de lo que las personas consideran placentero y generador de pensamiento creativo no está presente en los salones de clase actuales. Lo anterior, además de la carencia física ha creado una carencia conceptual en las personas que consideran que, estos elementos estéticos no son necesarios para el estímulo de la imaginación y del placer académico. Se crea así un hábito, es decir, una costumbre no consciente y errada de que el diseño de los espacios, el uso de elementos estéticos y didácticos no es necesario en el estímulo de la imaginación y el entendimiento del que hacer académico universitario.

Se hace necesario hacer un párrafo aparte en lo que respecta a las personas. Estas son sin duda el mayor estímulo estético, que desde los datos recogidos, tienen los estudiantes y profesores de la facultad de comunicación del Politécnico Colombiano Jaime Isaza Cadavid. Pero este estímulo estético que procuran las personas tiene algunas condiciones que no todas las personas las cumplen. Estas condiciones se refieren a la capacidad comunicativa, de innovación e intelectual, incluso, por encima de sus cualidades éticas. La novedad frente al conocimiento y la acción creativa es la mayor fuente de placer en el ámbito académico. Por eso las personas que procuran nuevos conocimientos o nuevas acciones creativas de aprendizaje son tenidas como generadoras de placer académico.

A pesar de lo anterior y de la poca o mucha presencia de espacios, acciones e incluso personas que sean fuente de placer académico y fuente de creatividad, las personas saben cómo regenerar la presencia del placer cuando 
sienten su ausencia. Provocar placer en el otro es la forma de garantizar placer personal al ser retribuido de esta misma forma por el otro. Así mismo, cuando se recibe un estímulo bello, ese mismo estímulo se devuelve al otro en una especie de danza entre cabeza y cola que potencia la experiencia estética al infinito. Así, la experiencia estética obtiene su propio placer al procurar placer al otro.

Los estímulos que se procura en los demás es el que por lo general se recibe del otro en una acción reacción binaria. Mas, esta reacción binaria puede ser triádica al obtener la intervención de la lógica racional la cual decide la mejor forma de responder desde la razón, llegando incluso, a transformar el tipo de signo estético de vuelta. De este modo, la producción de signos estéticos puede cambiar un estímulo negativo en uno positivo. Lo anterior demuestra además que la experiencia viva de la estética es dinámica del sujeto con su entorno y el signo que los media. El análisis individual de alguno de estos tres componentes es limitado y parcial para la comprensión de la experiencia.

El sujeto es también responsable de la estética viva del espacio de aprendizaje universitario en el sentido de que no solo percibe, sino que, interpreta $\mathrm{y}$ emite signos estéticos para ser leídos por los otros. Se teje así una comunidad estética responsable de lo que construye para sí mismo en la medida en que lo hace en el otro. Consecuente con esto no hay un dentro y un afuera del sujeto sino una pertenencia a una dinámica estética viva más compleja a la cual pertenece.
Consecuente con el concepto anterior de estética viva entre el sujeto, el entorno y sus signos, es el sujeto el que se convierte en el protagonista de esta triada porque su interpretación y la acción que da, esta, se pueda desprender como creencia de lo que debe ser y hacer, establece la diferencia entre todas las infinitas relaciones triádica existentes. Es decir, no todos responden de igual forma a un mismo estímulo, más, si se puede presuponer un tipo de reacción colectiva donde solo escapan aquellas reacciones mediadas por la lógica, la razón y la experiencia de cada sujeto.

\section{Referencias Bibliográficas}

Arguello, R. 1995. Estética y comunicación. Bogotá. Facultad de Ciencias sociales y Humanas, Universidad a Distancia.

Augustowsky, G. (2010). Las paredes del aula. El registro fotográfico en la investigación educativa. Conferencia pronunciada el 14 de noviembre de 2009. Escuela de Educación de la U de SA. Madrid, España. gñ

Baumgarten, A. 1999. Estética. Buenos Aires: Ed. Facultad de Filosofía y Letras. U.B.A.

Bedoya, Olga Lucia. (2014) Protocolo de análisis/Síntesis. Texto inédito sin publicar.

Duarte, J. (2003). Ambientes de aprendizaje: una aproximación conceptual. Estudios pedagógicos, No.29. pp. 97-113. Valdivia, Universidad Austral de Chile.

Eco, U. (1990). Arte y belleza en la estética medieval. Lima: Lumen.

Flexas, O. 2013. Apreciación estética de estímulos abstractos y figurativos: Datos conductuales y registros cerebrales. (Tesis de doctoral). Universidad de las islas baleares, España. 
García, M. y Alvira, F. (1994). El análisis de la realidad social métodos y técnicas de la investigación. Madrid: Alianza Editorial.

Gutiérrez, C. y Pérez, C. (2002). El espacio como elemento facilitador del aprendizaje. Una experiencia en la formación inicial del profesorado. Pulso, 25, pp. 133-146. Universidad de Alcalá, España.

Iris, P. (s.f.). Ambientes de aprendizaje. Una opción para mejorar la calidad de la educación (ensayo). Universidad Eurohisponoamericana. Veracruz, México.

Jiménez, Á. (2008). La escuela nueva y los espacios para educar. Proyecto de investigación en Arquitectura y pedagogía. Universidad del valle. Cali, Colombia.

Kant, I (1977). Crítica del juicio. Manuel García Morente (Traducción). Barcelona: Espasa-Calpe.

Moreno, G. y Molina, A. (1993). El ambiente Educativo. En: Planteamiento en educación. Intervención en planteamiento de planteamientos, Bogotá: Editorial.

Muñoz, G. (2016, septiembre 14). Mapas de sujeto. Maestría en Comunicación Educativa (clase magistral). Medellín Colombia. UTP-PCJIC.

Peirce, C. (1974). La ciencia de la semiótica. [Ebook] Buenos Aires: Ediciones Nueva Visión,

p.109. Recuperado de: http://mastor.cl/blog/ wp-content/uploads/2015/08/ PEIRCE-CH.-

S.-La-Ciencia-de-La-Semi\%C3\%B3tica. peirce/Peirce.html

Naranjo, J. y Torres, A. (1996). Ciudad educativa y pedagogías urbanas. Bogotá: Dimensión Educativa.

Nietzsche. F. 2012. El nacimiento de la tragedia. Andrés Sánchez Pascual (Traducción). España: Alianza editorial.
Robert, P. La educación en Finlandia. Los secretos de un éxito asombroso. Cada alumno es importante. Manuel Valdivia Rodríguez (Traducción). Colegio finlandés. Gard, Francia.

Suárez, M. y Garrido, M. (1989). Diseño espacial del aula de preescolar. Un estudio de caso. Colexio universitario de Ourense. España: Editorial. 